\title{
INVERSION OF BACKSCATTER IONOGRAMS INTO QUASI-PARABOLIC IONOSPHERIC LAYER PARAMETERS
}

\author{
S.N. Ponomarchuk \\ Institute of Solar-Terrestrial Physics SB RAS, \\ Irkutsk, Russia, spon@iszf.irk.ru
}

\author{
M.S. Penzin \\ Institute of Solar-Terrestrial Physics SB RAS, \\ Irkutsk,Russia,penzin@iszf.irk.ru
}

\begin{abstract}
We present an inversion scheme of the backscatter signal leading edge into parameters of the quasi-parabolic electron density profile, which is based on the comparison of experimental and calculated minimum delays of scattered signals with corresponding distances to the skip zone border. Input parameters are frequency dependences of minimum group path of signal propagation, derived from processing and interpreting backscatter ionograms. For a fixed sounding frequency, the ionospheric parameter pair - the critical frequency and the height of the F2-layer maximum - is defined as the intersection point of two curves representing solutions of minimization problems for discrep-
\end{abstract}

ancy functionals of the minimum group path and the distance to the skip zone border. Determining the ionospheric parameters by this inversion scheme on the sounding frequency grid allows us to construct a twodimensional distribution of electron density in the direction of backscatter sounding.

Keywords: ionosphere, ionogram, radio wave propagation, backscatter ionospheric sounding.

\section{INTRODUCTION}

The ionospheric backscatter sounding (BS) method has the greatest potential for the diagnostics of conditions of radio wave propagation along HF radio paths. A BS signal contains information about ionospheric conditions at a distance of several thousand kilometers from the observation site in any given direction. This significantly extends the capabilities of ionospheric stations of vertical sounding (VS) in studying the ionosphere and predicting its parameters. Inversion of BS ionograms is associated with solving the inverse problem, when quantitative ionospheric parameters are determined from BS data. The probing signal delays corresponding to the BS signal leading edge are used as characteristics to be measured. In fact, such inverse problems are classified as ill-posed, and finding stable solutions requires regularization methods. One of them is an approximation of true distribution of the electron density in the ionosphere by model functions whose parameters are to be determined [Rao, 1974; Norman, Dyson, 2006; Li et al., 2013]. To find a unique solution of the inverse problem for a given sounding frequency, we must vary the minimum group path with reference to the distance to the skip zone border. Such a reference can be made on the basis of the geometry of signal propagation along a path [Li et al., 2013].

The paper presents a scheme of inversion of the BS signal leading edge into parameters of electron density quasi-parabolic profile by comparing experimental and calculated minimum delays of scattered signals and respective distances to the skip zone border. Input parameters are the results of isolation of the BS signal leading edge, obtained after processing and interpreting BS ionograms [Penzin et al., 2019]. The BS signal leading edge identified by this method is, in fact, a scaled frequency dependence of the minimum group path of signal propagation calculated using the regular predictive ionospheric model without regard to random irregularities. Accordingly, it is only close to the true BS signal leading edge on the ionogram. The inversion scheme of BS ionograms has been realized for the spherically symmetric Earth - ionosphere waveguide with the electron density profile fitted by two quasi-parabolas with specified ratios of heights of the beginning of the layer and the joining point of the quasi-parabolas to the F2layer peak height. To determine the two unknown parameters of the profile - critical frequency and F2layer peak height - along with the minimum group path the distance to the skip zone border is varied, which allows us to find the unique solution. Unlike [ $\mathrm{Li}$ et al., 2013], we determine the required real distance to the skip zone border from the ratio of the minimum group path to the distance to the skip zone border, which is an adiabatic invariant, using results of simulation of BS signal characteristics [Penzin et al., 2019]. For a fixed sounding frequency, the pair of ionospheric parameters - critical frequency and F2-layer peak height — is defined as an intersection point of two curves, which are solutions of the problem of minimizing the discrepancy functional for the minimum group path and the distance to the skip zone border. The inversion scheme developed for BS ionograms is used for real-time calculation of ionospheric parameters from current BS data with the spatially distributed chirp ionosonde developed at ISTP SB RAS [Podlesnyi et al., 2013].

\section{MODEL OF THE ELECTRON DENSITY PROFILE}

We use a simplified formulation of the inverse problem. The electron density profile $N_{\mathrm{e}}(r)$, calculated by IRI [Bilitza et al., 2017], is approximated using two quasi-parabolas [Kurkin et al., 1981]: 


$$
\begin{aligned}
& N_{\mathrm{e}}(r)=N_{\mathrm{m}}\left\{\begin{array}{l}
d_{1}\left(1-r_{\mathrm{b}} / r\right)^{2}, r_{\mathrm{b}}<r<r_{0}, \\
1-d_{2}\left(r_{\mathrm{m}} / r-1\right)^{2}, r_{0}<r<r_{\mathrm{m}},
\end{array}\right. \\
& d_{1}=\left[\left(1-r_{\mathrm{b}} / r_{0}\right)\left(1-r_{\mathrm{b}} / r_{\mathrm{m}}\right)\right]^{-1}, \\
& d_{2}=\left[\left(r_{\mathrm{m}} / r_{\mathrm{b}}-1\right)\left(r_{\mathrm{m}} / r_{0}-1\right)\right]^{-1} .
\end{aligned}
$$

This approximation of $N_{\mathrm{e}}(r)$ as two quasi-parabolas describes the vertical distribution of the electron density for the F2 layer. Four parameters defining the two quasi-parabolas - the radial coordinate of the beginning of the layer $r_{\mathrm{b}}$, the coordinate of the profile maximum $r_{\mathrm{m}}$, the joining point of quasi-parabolas $r_{0}$, and the electron density at the layer maximum $N_{\mathrm{m}}$ - are determined from $N_{\mathrm{e}}(r)$, using the least square method with the twodimensional version of the bisection method. The profile $N_{\mathrm{e}}(r)$ is calculated by IRI [Bilitza et al., 2017]. For the quasi-parabolic ionospheric layer model, HF radio wave propagation characteristics can be calculated from analytical expressions for integrals [Croft, 1968; Kurkin et al., 1981]. In the proposed inversion scheme for BS ionograms, two parameters of quasi-parabolas are identified - the critical frequency $f_{\text {cr }}=\sqrt{80.6 N_{\mathrm{m}}}$ and the F2-layer peak height $h_{\mathrm{m}}=r_{\mathrm{m}}-a$, where $a$ is the Earth radius. The other two parameters - the height of the beginning of the layer $h_{\mathrm{b}}=r_{\mathrm{b}}-a$ and the height of the joining point of the quasi-parabolas $h_{0}=r_{0}-a-$ are related to $h_{\mathrm{m}}$ by the ratios $h_{\mathrm{b}}=\frac{k_{\mathrm{b}}-1}{k_{\mathrm{b}}} h_{\mathrm{m}} \quad$ and $h_{0}=\frac{k_{0}-1}{k_{0}} h_{m}$. The coefficients $k_{\mathrm{b}}$ and $k_{0}$ are found from the condition of the best approximation of the $N_{\mathrm{e}}(r)$ profile as two quasi-parabolas.

\section{INTERPRETATION OF IONOGRAMS}

An input parameter in the inverse problem is a delay array corresponding to the leading edge of received signals on a BS ionogram. To identify the BS signal leading edge in an ionogram, Ponomarchuk et al. [2018] and Penzin et al. [2019] have proposed a method of automatic processing and interpretation of ionospheric backscatter sounding data using a continuous chirp signal. Processing of experimental ionograms is based on filtering of raw data with their subsequent compression through cellular automaton. The ionogram interpretation technique relies on results of simulation of BS signal characteristics on the leading edge in the context of the waveguide approach [Ponomarchuk et al., 2009; Penzin et al., 2019] with IRI [Bilitza et al., 2017]. The BS signal leading edge for a frequency $f$ corresponds to the minimum scattered signal propagation delay, or to the minimum group path $P_{\mathrm{m}}$. Therefore, the leading edge on a BS ionogram can be identified by calculating the frequency dependence of the minimum group path $P_{\mathrm{m}}(f)$ for the oblique sounding (OS) signal. In the waveguide approach, trajectory characteristics of signals - group path of signal propagation along a trajectory, angles of signal departure from a transmitter point and arrival at a receiver point - are calculated from the stationarity equation, which defines the central number $n$ for a group of phased normal modes. For a ground-based transmitter and receiver, it has the form

$$
\Delta \Phi_{n}=\left(\Phi_{n}-\Phi_{n+1}\right)=2 \pi l
$$

where $l$ is the number of signal trajectory reflections from the ionosphere, $\Phi_{\mathrm{n}}=k a \int_{0}^{\theta} \gamma_{\mathrm{n}}\left(\theta_{1}\right) d \theta_{1}$ is the phase of the normal mode [Kurkin et al., 1981]. The angle of trajectory departure $\Delta$ from the transmitter point is related to the central number $n$ of the group of phased normal modes by $\cos \Delta=\gamma_{n}$, where $\gamma_{n}$ is the spectral parameter of the normal mode [Kurkin et al., 1981]. In the spherically symmetric waveguide, the stationarity equation reduces to an expression for the jump distance along Earth's surface through the connection between the elevation angle $\Delta$ and the spectral parameter $\gamma_{n}$ :

$$
D=2 a^{2} \cos \Delta \int_{a}^{r_{n}} \frac{d r}{r \sqrt{\varepsilon r^{2}-a^{2} \cos ^{2} \Delta}} .
$$

Here, $\varepsilon=1-\frac{80.6 N_{\mathrm{e}}(r)}{f^{2}}$ is the cold plasma permittivity [Kurkin et al., 1981], $r_{n}$ is the trajectory reflection point in the ionosphere. Solving transcendental stationarity equation (2) for the angular distance $\theta$ for given values $n_{i}$ allows us to construct an analog of the variation of range with angle - the dependence $D\left(n_{i}\right)=a \theta\left(n_{i}\right)$. By calculating the group delay of the central mode $\tau_{n_{i}}$ and using $\theta\left(n_{i}\right)$, obtain an analog of the variation of group path with angle - the dependence $P\left(n_{i}\right)$, where $P$ is the group path. Figure 1 plots $D(\Delta)$ (blue line) and $P(\Delta)$ (red line) of a one-hop signal for an operating frequency $f=10 \mathrm{MHz}$ for the spherically symmetric Earthionosphere waveguide. Values of the central number $n_{i}$ are converted into ray departure angles $\Delta_{i}$ through the connection between $n_{i}$ and $\gamma_{n}$, defined by the equation for the spectrum of normal modes [Kurkin et al., 1981]. $D(\Delta)$ and $P(\Delta)$ have been calculated for the electron density profile $N_{\mathrm{e}}(r)$, approximated using two quasiparabolas. Figure 2 shows the plasma frequency profile $f_{\mathrm{e}}(h)=\sqrt{80.6 N_{\mathrm{e}}(h)}$ calculated by IRI (black line), and the plasma frequency profile $f_{\mathrm{e}}(h)$ as two quasiparabolas (red line) with connection coefficients of the parameters $h_{\mathrm{b}}$ and $h_{0}$ with $h_{\mathrm{m}}: k_{\mathrm{b}}=1.5$ and $k_{0}=8$.

For the given frequency $f$ and distance $D$ there are two solutions of stationarity equation (2) for the angle of trajectory departure from a transmitter point, which correspond to low and high rays [Davies, 1969]. In Figure 1, vertical dashed lines indicate solutions for $f=10 \mathrm{MHz}$ and $D=1100$ km for the F2 channel: $\Delta_{1}$ - low ray, $\Delta_{2}$ - high ray. With decreasing range at a fixed frequency $f$, the high ray merges to the low ray at the minimum point of $D(\Delta)$. The minimum point is that of the skip zone border $D_{\mathrm{m}}$.

The operating frequency $f$ for $D_{\mathrm{m}}(f)$ is the maximum usable frequency $f_{\mathrm{m}}$ (MUF) of radio communication. A minimum in $P(\Delta)$ is determined by the minimum group path $P_{\mathrm{m}}$ [Davies, 1969]. Figure 1 shows $D_{\mathrm{m}}$ and $P_{\mathrm{m}}$ for the F2 channel. We can see that minima in $P(\Delta)$ and $D(\Delta)$ 


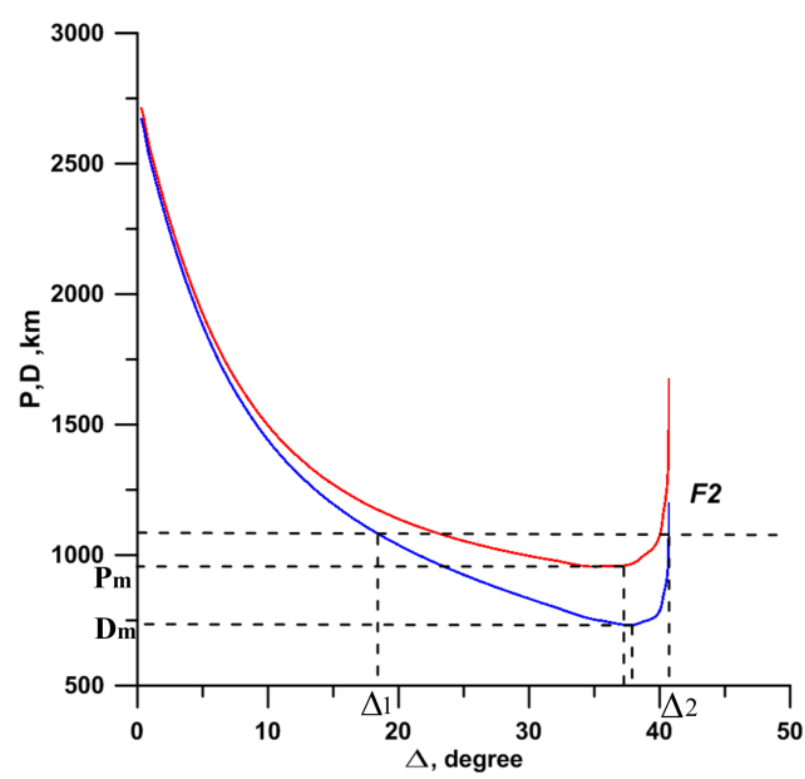

Figure 1. $D(\Delta)$ and $P(\Delta)$

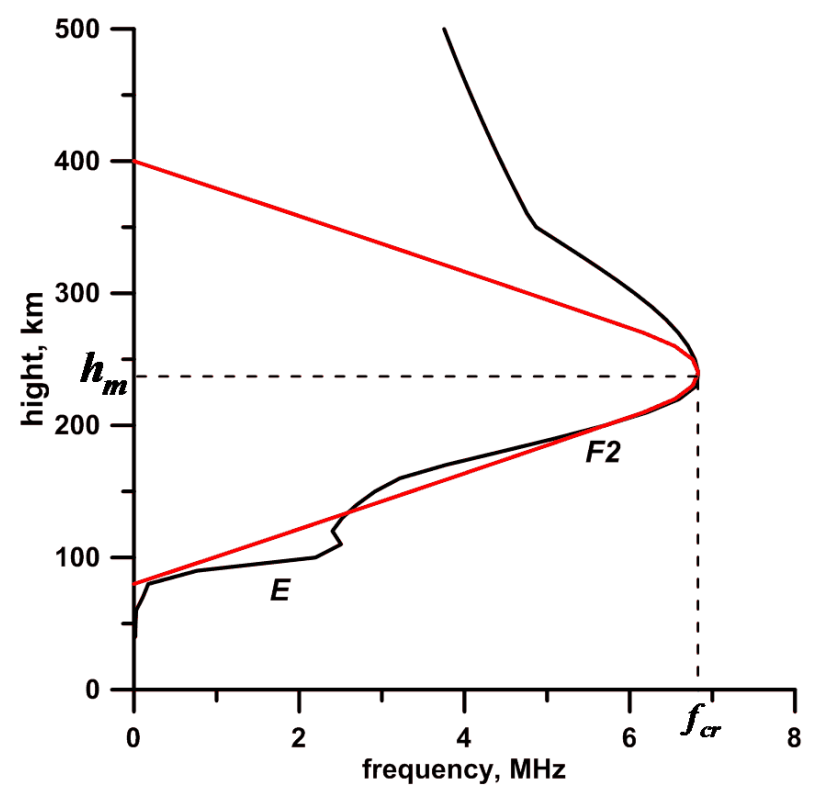

Figure 2. Plasma frequency profile $f_{\mathrm{e}}(h)$

correspond to different angles of trajectory departure, therefore the distance to the skip zone border $D_{\mathrm{m}}$ does not coincide with the distance at which the group path is minimum $P_{\mathrm{m}}$. As the operating frequency increases, this difference decreases.

Figure 3 displays a BS experimental ionogram obtained with the ISTP SB RAS chirp ionosonde [Ponomarchuk et al., 2017]. A transmitter of BS signals is in the town of Usolye Sibirskoye $\left(52.88^{\circ} \mathrm{N}, 103.26^{\circ} \mathrm{E}\right)$. A receiver is in the village of Tory, the Republic of Buryatia $\left(51.70^{\circ} \mathrm{N}, 103.0^{\circ} \mathrm{E}\right)$. The radiation azimuth is $55^{\circ}$. Time of reception - November 18, 2005, 03:40 UT. Figure 4 presents the results of processing and simulation of the minimum group path and the skip zone border, the results of interpretation of BS ionograms. Blue dots mark high-amplitude BS signals identified during secondary processing; the solid green line indicates results of simulation of the frequency dependence of the minimum group path $P_{\mathrm{m}}$ of the propagation mode $1 \mathrm{~F} 2$;

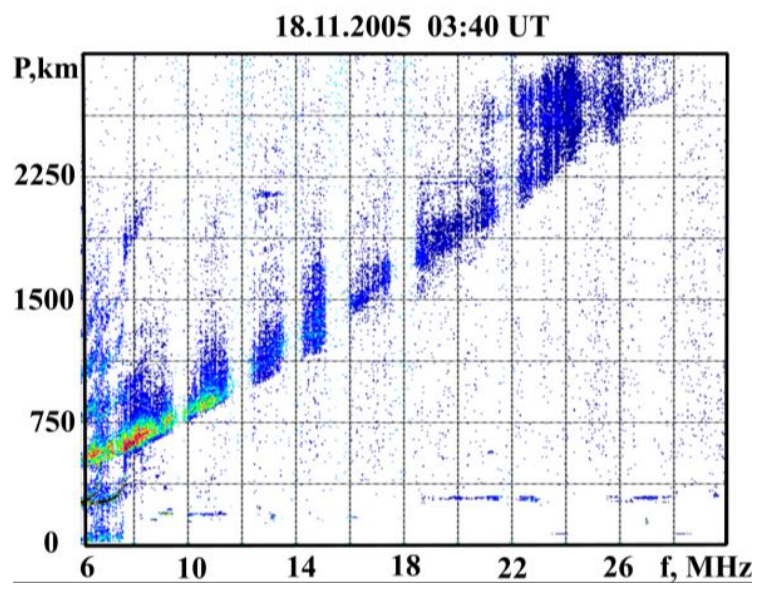

Figure 3. BS ionogram for November 18, 2005, 03:40 UT

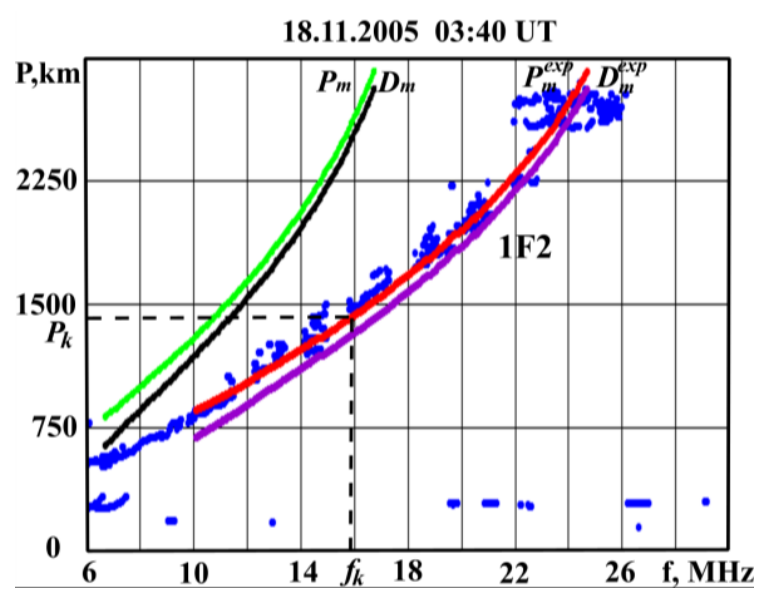

Figure 4. Results of processing and interpretation of the BS ionogram for November 18, 2005, 03:40 UT

the black line is the results of simulation of the distance to the skip zone border $D_{\mathrm{m}}$.

BS signal characteristics were simulated by solving stationarity equation (2), using electron density profiles calculated by IRI. The input parameter of the model is the solar activity index $F 10.7$. The solid red line represents the results of interpretation of BS signals $P_{\mathrm{m}}^{\exp }$, arriving at the receiver point after single reflection from the $\mathrm{F} 2$ layer. The frequency dependence $P_{\mathrm{m}}^{\exp }(f)$ is in fact scaled $P_{\mathrm{m}}(f)$ and accordingly close to the BS signal leading edge on the ionogram. Identifying the signal leading edge $P_{\mathrm{m}}^{\exp }(f)$ allows us to calculate the respective frequency dependence of the skip zone border $D_{\mathrm{m}}^{\exp }(f)$ from the adiabatic ratio $P_{m} / D_{m}$ at a relative frequency grid $v=f / \bar{f}_{\mathrm{m}}$, which changes little with ionospheric parameter variations [Ponomarchuk et al., 2018; Penzin et al., 2019]. Here, $\bar{f}_{\mathrm{m}}$ is the maximum usable frequency for the maximum hop distance. For the given group path $P_{k}$, we calculate $\eta=P_{k} / D_{\mathrm{m}}$, using $P_{m}(f)$ and $D_{m}(f)$ (see Figure 4). Further, from $P_{k}$ on the real $P_{\mathrm{m}}^{\exp }(f)$ we determine $f_{k}$ and the corresponding distance to the skip zone border $D_{k}^{\exp }=P_{k} / \eta$. In Figure 4, the frequency dependence $D_{\mathrm{m}}^{\text {exp }}(f)$ is marked with the purple line. 


\section{INVERSION OF IONOGRAMS}

The inversion scheme of the BS signal leading edge to determine the ionospheric parameters $\left(f_{\mathrm{cr}}, h_{\mathrm{m}}\right)$ consists of three stages.

1. On frequency grid $f_{k}, k=\overline{1, N}$, functions $D_{\mathrm{m}}^{k}\left(f_{\mathrm{cr}}, h_{\mathrm{m}}\right)$ and $P_{\mathrm{m}}^{k}\left(f_{\mathrm{cr}}, h_{\mathrm{m}}\right)$ are calculated for different admissible pairs of ionospheric parameters $\left(f_{\text {cr }}\right.$, $\left.h_{\mathrm{m}}\right)$ with quasi-parabolic dependence of profile $N_{\mathrm{e}}(r)$. Parameters of the quasi-parabolas $h_{\mathrm{b}}$ and $h_{0}$ are determined from $h_{\mathrm{m}}$ according to the specified coefficients $k_{\mathrm{b}}$ and $k_{0} . D_{\mathrm{m}}^{k}\left(f_{\mathrm{cr}}, h_{\mathrm{m}}\right)$ and $P_{\mathrm{m}}^{k}\left(f_{\mathrm{cr}}, h_{\mathrm{m}}\right)$ are computed by solving transcendental stationary equation (2) within the Earth - ionosphere spherically symmetrical waveguide.

2. From the given frequency $f_{k}$, we determine the group path $P_{k}$ using $P_{\mathrm{m}}^{\exp }(f)$, and the distance $D_{k}$ to the skip zone border using $D_{\mathrm{m}}^{\exp }(f)$.

3. A pair of ionospheric parameters $\left(f_{\mathrm{cr}}, h_{\mathrm{m}}\right)_{k}$ is determined from equalities

$$
\begin{aligned}
& D_{k}=D_{\mathrm{m}}^{k}\left(f_{\mathrm{cr}}, h_{\mathrm{m}}\right), \\
& P_{k}=P_{\mathrm{m}}^{k}\left(f_{\mathrm{cr}}, h_{\mathrm{m}}\right)
\end{aligned}
$$

as the intersection point of two curves that are solutions of (5) and (6) with given $D_{k}$ and $P_{k}$.

Figure 5 shows the surface $D_{\mathrm{m}}^{k}\left(f_{\mathrm{cr}}, h_{\mathrm{m}}\right)$ for $f_{k}=16$ $\mathrm{MHz}$ with the line of the solution of (5) with the corresponding distance $D_{k}=1329.12 \mathrm{~km}$ (see Figure 4). Figure 6 depicts the surface $P_{\mathrm{m}}^{k}\left(f_{\mathrm{cr}}, h_{\mathrm{m}}\right)$ for $f_{k}=16$ $\mathrm{MHz}$ with the line of solution of (6) with $P_{k}=1436.87$ $\mathrm{km}$. Figure 7 presents solutions of (5) and (6) for $f_{k}=16 \mathrm{MHz}$ with $D_{k}=1329.12 \mathrm{~km}$ and $P_{k}=1436.87 \mathrm{~km}$ on the plane $\left(f_{\mathrm{cr}}, h_{\mathrm{m}}\right)$.

Figure 8 exemplifies time variations of $f_{\mathrm{cr}}$ and $h_{\mathrm{m}}$ at a distance of $1500 \mathrm{~km}$ from the receiver point, obtained from the results of inversion of BS ionograms, taken with the chirp ionosonde in November 2005. The radiation azimuth is $55^{\circ}$. Time of reception - November 18, 2005, 00:00-08:00 UT. The red line indicates results of BS ionogram inversion; the blue line, IRI calculation results; the black line, experimental values of parameters obtained from VS ionograms at the ionospheric station Yakutsk $\left(62^{\circ}, 129.7^{\circ}\right)$.

The skip zone border corresponding to $D_{k}$ is formed by the ionospheric region at a distance $D_{k} / 2$ from the transmitter, as inferred from the geometry of the caustic line in the waveguide for downward rays of one-hop propagation mode. Below the caustic line is the skip zone. Therefore, calculating the ionospheric parameters on the frequency grid $f_{k}, k=\overline{1, N}$ with respective $P_{k}$ and $D_{k}$ enables the construction of a two-dimensional electron density distribution in the BS direction.

\section{CONCLUSION}

In conclusion, let us note the main differences in our scheme of inversion of the BS signal leading edge into parameters of the quasi-parabolic ionospheric F2 layer from already-known inversion schemes.

1. To obtain a unique solution of the inverse problem, not only the minimum group path is varied but also the distance to the skip zone border. Finding the real distance to the skip zone border using the identified BS signal leading edge is the main element in the proposed scheme of inversion of BS ionograms into ionospheric parameters.

2. Another novel element of the scheme is the use of preliminary calculations of the minimum group path and the distance to the skip zone border as a function of two variables - the critical frequency and the F2-layer peak height for different sounding frequencies - which allows us to utilize the this scheme in real time.

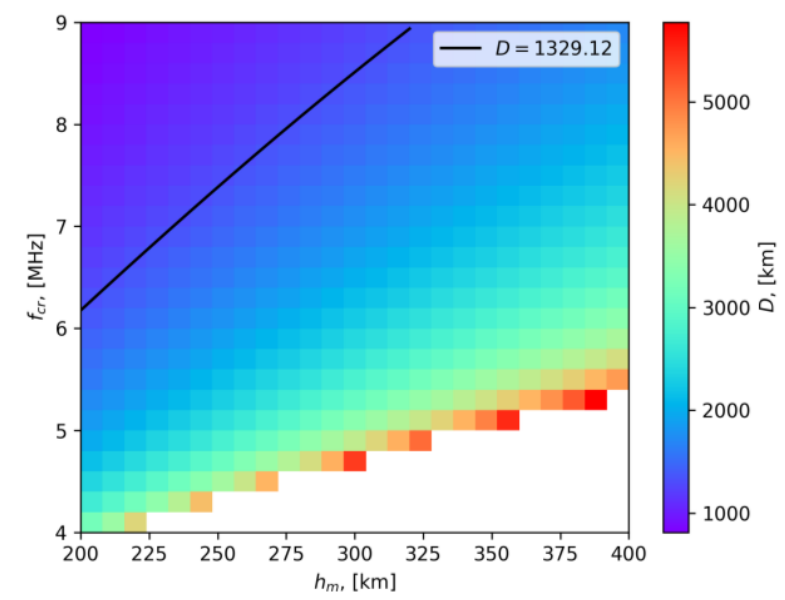

Figure 5. Surface $D_{\mathrm{m}}^{k}\left(f_{\mathrm{cr}}, h_{\mathrm{m}}\right)$

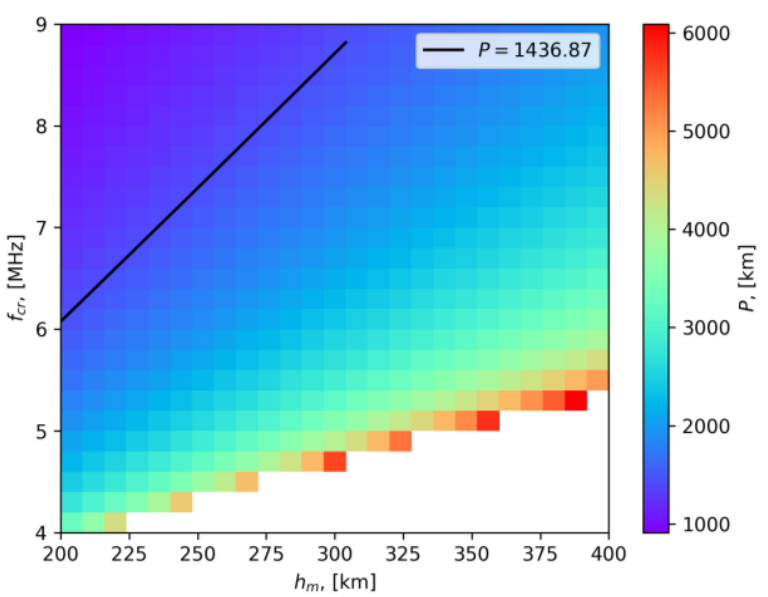

Figure 6. Surface $P_{\mathrm{m}}^{k}\left(f_{\mathrm{cr}}, h_{\mathrm{m}}\right)$ 


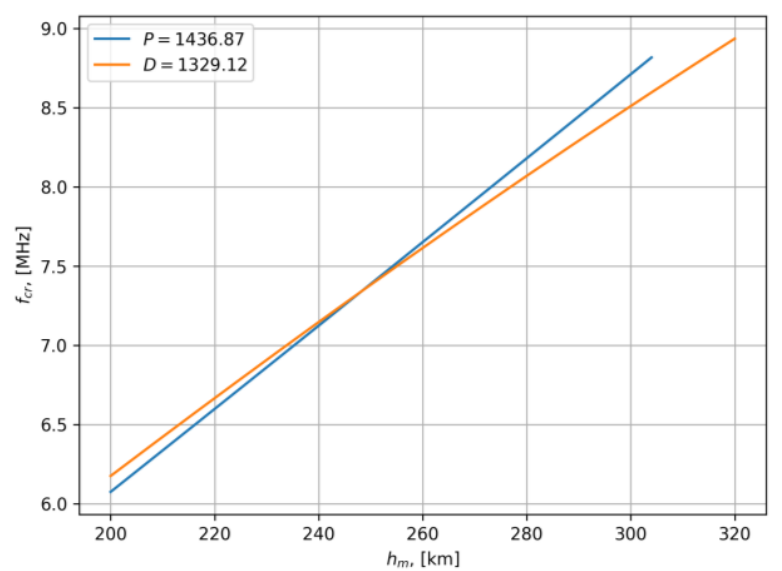

Figure 7. Solutions of combined equations (5) and (6) for $f_{k}=16 \mathrm{MHz}$ with $D_{k}=1329.12 \mathrm{~km}$ and $P_{k}=436.87 \mathrm{~km}$
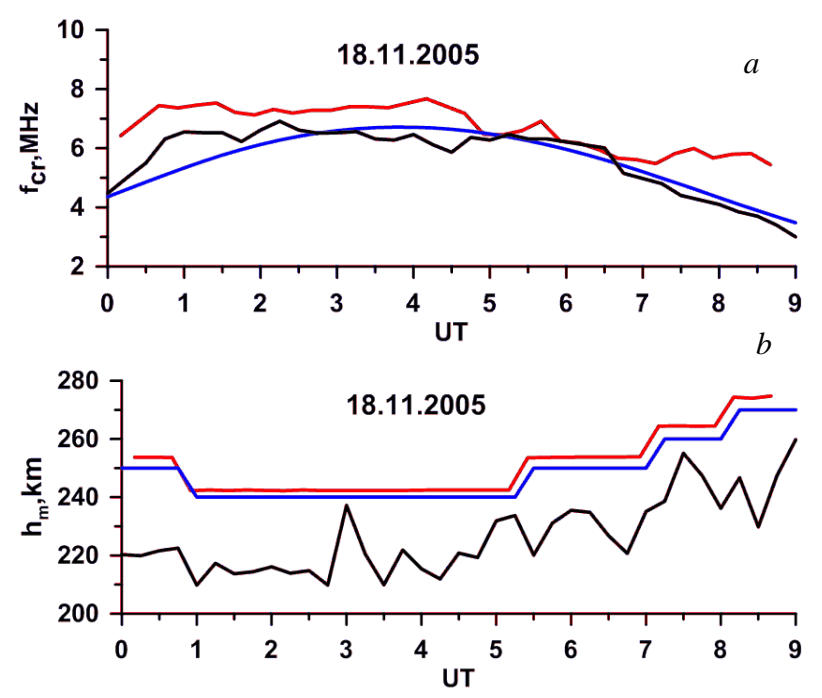

Figure 8. Temporal variations of F2-layer parameters at a distance of $1500 \mathrm{~km}$ from the transmitter point: $a$ is the critical frequency $f_{\text {cr }}, b$ is the peak height $h_{\mathrm{m}}$

Also note that the accuracy in determining the ionospheric parameters in our scheme of inversion of BS ionograms depends directly on the result of identification of the BS signal leading edge, used to recover the frequency dependence of the distance to the skip zone border.

In the future, we plan to extend this scheme to the ionospheric E and F1 layers.

The work was performed with budgetary funding of Basic Research program II.12 and was supported by RFBR grant No. 19-02-00513-a. The experimental data was obtained using the equipment of Center for Common Use «Angara» [http://ckp-rf.ru/ckp/3056].

\section{REFERENCES}

Benito E., Bourdillon A., Bourdillon A., Rannou V. Inversion of HF backscatter ionograms using elevation scans. $J$. Atm. Solar-Terr. Phys. 2008, vol. 70, no. 15, pp. 1935-1948. DOI: 10.1016/j.jastp.2008.09.031.

Bilitza D., Altadill D., Truhlik V., Shubin V., Galkin I., Reinisch B., Huang X. International Reference Ionosphere 2016: From ionospheric climate to real-time weather predic- tions. Space Weather. 2017, vol.15, no. 2, pp. 418-429.

Croft T.A. Hoogansian H. Exact ray calculations in a quasi-parabolic ionosphere with no magnetic field. Radio Sci. 1968, vol. 3, no. 1, pp. 69-74.

Davies K. Ionospheric Radio Waves. Blaisdell, London, 1969. $460 \mathrm{p}$

Kurkin V.I., Orlov I.I., Popov V.N. Metod normalnykh voln $v$ problem korotkovolnovoi svyazi [Normal Wave Technique in HF Radio Communication Problem]. Moscow, Nauka, 1981. (In Russian).

Li N., Zhao Z., Zhou C., Chen G., Yang G., Huang S., Li T. Inversion of sweep frequency backscatter ionogram from Monostatic HF Sky-Wave Radar. IEEE Geoscience and Remote Sensing Lett. 2013, vol. 10, no. 6, pp. 1360-1364.

Norman R.J., Dyson P.L. HF radar backscatter inversion technique. Radio Sci. 2006, vol. 41, RS4010. DOI: 10.1029/2005RS003355.

Penzin M.S., Ponomarchuk S.N., Grozov V.P., Kurkin V.I. Real-time techniques for interpretation of ionospheric backscatter sounding data. Radio Sci. 2019, vol. 54, iss. 5, pp. 480-491. DOI: 10.1029/2018RS006656.

Podlesnyi A.V., Brynko I.G., Kurkin V.I., et al. Multifunctional chirp ionosonde for monitoring the ionosphere. Geliogeofizicheskie issledovaniya [Heliogeophys. Res.]. 2013, no. 4, pp. 24-31. (In Russian).

Ponomarchuk S.N, Kurkin V.I., Oinats A.V. The diagnostics of ionosphere and earth ground surface by backscatter sounding data. Proc. PIERS-2009. Moscow, 2009. vol. I-II, pp. 1307-1310.

Ponomarchuk S.N., Grozov V.P., Kotovich G.V., Kurkin V.I., Penzin M.S. Diagnostics of HF radio channel: based on data from backscatter ionospheric sounding by continuous chirp signal. Solar-Terrestrial Physics. 2018, vol. 4, iss. 2. pp. 17-23. DOI: 10.12737/stp-42201804.

Ponomarchuk S.N., Kurkin V.I., Penzin M.S. Features of backscatter ionospheric sounding as studied with a chirp ionosonde. Solar-Terr. Phys. 2017, vol. 3, iss. 3, pp. 54-60. DOI: 10.12737/stp-332017062017.

Rao N.N. Inversion of sweep-frequency sky-wave backscatter leading edge for quasiparabolic ionospheric layer parameters. Radio Sci. 1974, vol. 9, no. 10. pp. 845-847.

How to cite this article

Ponomarchuk S.N., Penzin M.S. Inversion of backscatter ionograms into quasi-parabolic ionospheric layer parameters. SolarTerrestrial Physics. 2020. Vol. 6. Iss. 3. P. 67-71. DOI: 10.12737/stp63202009. 